\title{
Multi-Agent Collaborative Control Method of Ramps Based on Fuzzy Neural Network
}

\author{
Niu Zhonghai \\ School of Traffic and Transportation \\ Beijing Jiaotong University \\ Beijing, China \\ e-mail: 09114195@bjtu.edu.cn \\ Jia Yuanhua \\ School of Traffic and Transportation \\ Beijing Jiaotong University \\ Beijing, China \\ e-mail: yhjia@bjtu.edu.cn
}

\author{
Chen Feng \\ Signal \& Communication Research Institute \\ China Academy of Railway Sciences \\ Beijing, China \\ e-mail: 08114212@bjtu.edu.cn \\ Zhang Liangliang \\ School of Traffic and Transportation \\ Beijing Jiaotong University \\ Beijing, China \\ e-mail: 10114212@bjtu.edu.cn
}

\begin{abstract}
In order to improve the growing serious traffic congestion on the junction of freeway and expressway (junction of road network in short), starting from the on-ramp metering, considering the main line and its on-ramp control requirements, the multi-agent collaborative was introduced. And the relativity of different segments of junction of road network was analyzed. Meanwhile, the correlation of different segments was calculated. With the consistency of control objectives for urbanized segments of expressway, a relative density model of multi-agent consistency was proposed. Then, on basis of multi-agent and fuzzy control theory, a multi-agent ramp cooperative control model based on fuzzy neural network was presented. Urbanized segments of BeijingTianjin-Tanggu Expressway were selected to validate the model. The results show that the model was effective. The method can stabilize the density of the urbanized expressway, and relief traffic congestion in the area.
\end{abstract}

Keywords-expressway; cooperative control; ramp metering; fuzzy neural network; multi-agent

\section{INTRODUCTION}

Demand of car transportation rapidly increases with economic and social development of cities. Thus, traffic congestion is becoming serious in cities, even spreading to surrounding suburbs. Control of traffic congestion has become an important social issue facing large and mediumsized cities, especially in urbanized segments of expressway. Increasing demands for city-suburb commuting, intercity travel and holiday travel lead to rising traffic loads in urbanized segments of expressway, with frequent traffic jams.

Currently, A. Kotsialos and M.Papageorgiou strategies and methods of integrated and cooperative control for road network; they advocated system optimization should be replaced with user optimization ${ }^{[1,2]}$. I. Papamichail proposed a three-tier hierarchical control structure; meanwhile, multiramp heuristic cooperative control strategies and methods based on MPC were studied ${ }^{[3]}$. J. Li established collaborative network flow equations under dynamic impedance balanced conditions, and developed control strategies for virtual control simulation of actual objects ${ }^{[4]}$. J. $\mathrm{K}$. He proposed on-ramp controller with simulation based on BP neural network; the results showed that the model had good control effect ${ }^{[5]}$. X. P. Fan proposed fuzzy neural network controller based on GA-BP, with detailed design for the controller; road traffic density was well stabilized by the controller ${ }^{[6]}$. G. Chai adopted the Greenshields model to determine influence scope of traffic congestion; density was used as control variable for traffic optimization through local and coordinating regulation rates ${ }^{[7]}$. L. Wang designed a multi-signal self-adaptive control method to reduce queue length of exit ramp and queuing overflow of downstream intersection ${ }^{[8]}$. J. C. Rao established exit ramp regional model based on improved cell transmission model, with verification using traffic data generated by simulation software ${ }^{[9]}$. A. Fares introduced a new microscopic framework at the network level and an associated cooperative Q-learning algorithm, and proposed a multiagent reinforcement learning control system for ramp metering ${ }^{[10]}$. In the work, advantages of fuzzy control and neural network were integrated in fuzzy neural network. Self-learning ability of neural networks was well exerted with full use of expert experiences; meanwhile, fuzzy rules and membership functions were adjusted according to changes of outside world, thus forming more reasonable and accurate reasoning structure. Multi-agent consistency and correlation of road expressway was analyzed based on fuzzy neural network, designing cooperative control method of ramps. 


\section{ANALYSIS OF ASSOCIATED RAMPS IN SAME ROAD}

Cooperative control among multi ramps was involved in the same road. The control objectives were consistent among multi ramps. Through ramp control, density and flow or velocity of road was controlled within a reasonable range, ensuring smooth operation of road traffic, with less traffic jams in rush hour. Fig. 1 showed that associated multi ramps were divided into different sections. Each section was regarded as an agent control unit. The control units were arranged linearly in the main line. Control units were directly correlated with adjacent ones, and indirectly correlated with other control units in positive and negative directions; the correlation declined with the distances among control units. Correlations or delayed correlations of traffic flow between different control units were calculated. Considering complexity of distributed network size, the range of ramp number was determined as 1-6 in positive and negative directions, according to the obtained relevance. Further range of ramps had weak correlations, with increasing complexity and resource consumption of distributed multi-agent algorithm. In this case, structure of ramp cooperative control in the same road was considered as multi-agent consistency control structure consisting of different units.

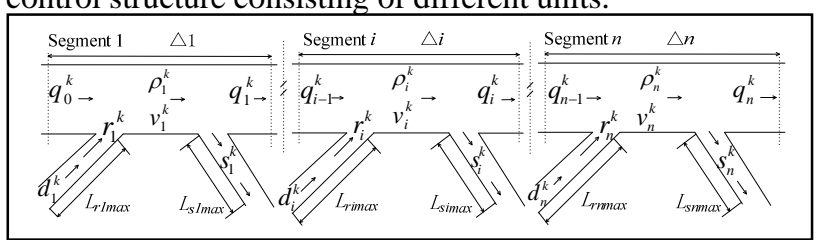

Figure 1. Typical shape of continuous segments and connected multiramp

\section{COOPERATIVE CONTROL METHOD BASE ON FUZZY MULTI- AGENT}

\section{A. Relative density model based on multi-agent consistency}

Multi-agents had consistent objective—controlling road at desired density. However, traffic flow data of segments were relevant for different agents, thus single-agent control unit was difficult to achieve overall optimal control. Therefore, the interactions among different segments were considered in the work. The range of ramp number in positive and negative directions was determined according to the correlation. Based on traffic conditions of segments for directly and indirectly adjacent agents, relative density function of multi-agent control consistency was constructed with actual density $\rho(k)$ and desired density $\rho_{\mathrm{d}}(k)$ of main line. Relative density $\rho^{\circ}(k)$ of the main line was calculated, and fed back to agent control unit of fuzzy neural network. Relative density $\rho^{\circ}(k)$ was used as the input of actual density $\rho(k)$ to achieve multi-ramp cooperative control.

Density vector $\rho_{i}$ was supposed as reference sequence, while $\rho_{j}$ was comparative sequence:

$$
\rho_{i}=\left\{\rho_{i}(1), \rho_{i}(2), \cdots, \rho_{i}(k)\right\}
$$

Correlation functions:

$$
\rho_{i}=\left\{\rho_{i}(1), \rho_{i}(2), \cdots, \rho_{i}(k)\right\}
$$

$$
R_{i j}=\frac{\sum_{i, j=1}^{k}\left(\rho_{i}-\bar{\rho}_{i}\right)\left(\rho_{j}-\bar{\rho}_{j}\right)}{\sqrt{\sum_{i, j=1}^{k}\left(\rho_{i}-\bar{\rho}_{i}\right)^{2} \cdot\left(\rho_{j}-\bar{\rho}_{j}\right)^{2}}}
$$

Through correlation analysis, the number of direct and indirect agents in positive and negative directions was $m$ and $n$. It was supposed that $l$ the control distance of different agents; $\alpha$ the pass parameter in positive direction; $\beta$ the pass parameter of negative direction. Therefore, it was obtained:

Pass density in positive direction was

$$
\rho_{+}^{\prime}(k)=\partial \cdot\left(\sum_{i=2}^{m} \frac{R_{0 i}(k)}{\sum_{i=2}^{m} R_{0 i}(k)} \cdot \frac{l_{i}(k)}{\sum_{i=2}^{m} l_{i}(k)}\left(\rho_{i}(k)-\rho_{d}(k)\right)\right)
$$

Pass density in negative direction was

$$
\rho_{-}^{\prime}(k)=\beta \cdot\left(\sum_{j=2}^{n} \frac{R_{0 j}(k)}{\sum_{i=2}^{n} R_{0 j}(k)} \cdot \frac{l_{j}(k)}{\sum_{j=2}^{m} l_{j}(k)}\left(\rho_{j}(k)-\rho_{d}(k)\right)\right)(5)
$$

Total pass density was

$$
\rho^{\prime}(k)=\rho_{+}^{\prime}(k)+\rho_{-}^{\prime}(k)
$$

Relative density of road consistency was

$$
\begin{aligned}
& \rho^{\circ}(k)=\rho(k), \rho^{\prime}(k) \leq 0 \\
\rho^{\circ}(k)= & \rho(k)+\rho^{\prime}(k), \rho^{\prime}(k) \geq 0
\end{aligned}
$$

Relative density of road was adopted as input variable of actual density in control model of single ramp agent. The regulation rate of single-agent consistency was calculated.

\section{B. Agent control model based on fuzzy neural network}

Fuzzy neural network properly combined fuzzy logic and neural network with their advantages. It can be applied to express vague or qualitative knowledge, with abilities of parallel computing, distributed information storage, strong fault tolerance and self-adaptive learning. In the work, fuzzy neural network control model was selected as agent control units. The basic structure of single-ramp control model based on fuzzy neural network consisted of input layer, fuzzification layer, layer of fuzzy rules, defuzzification layer and output layer.

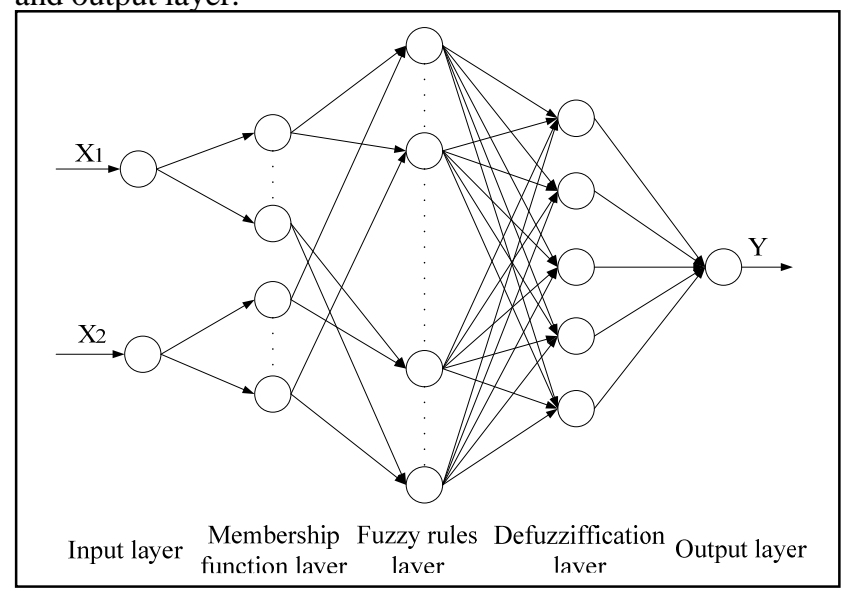

Figure 2. Structure of single-ramp control model 
It was supposed that $x_{i}^{k}$ was the $i$-th input of the $k$-th layer, and $y_{j}^{k}$ the $j$-th node output of the $k$-th layer. Therefore, fuzzy neural network had five layers as follows.

The first layer was input layer. Parameters of expressway traffic flow were used as input values. The layer only contained two neurons $x_{1}^{1}$ and $x_{2}^{1}$ reflecting traffic states of road and ramp, respectively. Input variables directly entered the next layer, with connection factor of 1 .

The second layer was membership function. Traffic states of expressway and ramp were fuzzified, calculating the ratios of actual traffic flow to traffic capacity. The traffic states after fuzzification included $\{N B, N M, N S, Z O, P S, P M$, $P B\}$ in descending order. Membership function adopted Gaussian function. The layer contained 14 neurons representing 14 traffic states of expressway and ramp. The neurons were two-input fuzzy sets; the input was fuzzy value of input variables, while the output was responsive membership.

$$
y_{j}^{2}=\exp \left(-\frac{\left(x_{j}^{2}-m_{i j}\right)^{2}}{\sigma_{i j}^{2}}\right)
$$

where, $m_{i j}$ and $\sigma_{i j}$ were the mean and standard of Gaussian membership function for the $i$-th input variable and j-th fuzzy set, respectively.

The third layer was fuzzy rule with 49 neurons. Each neuron represented a rule of fuzzy inference system. Input value of the $j$-th neuron was linguistic variable value of $I F$ in the $j$-th rule, while the output was fitness of the $j$-th rule.

$$
y_{j}^{3}=x_{1}^{3} \cdot x_{2}^{3}
$$

where $y_{j}^{3}$ was fitness of the $j$-th rule.

The fourth layer was defuzzification. There were 5 neurons corresponding to 5 fuzzy sets of the output. Connecting lines corresponded to fuzzy rules table in the third layer; $\mathrm{R}$ inputs of the $i$-th neuron was $a_{r}(r=1,2, \cdots, 49)$.

$$
y_{j}^{4}=\min \left(1, \sum_{r=1}^{49} a_{r}\right)
$$

The fifth layer was output layer with only one neuron. Its input was output $y_{j}^{4}$ of the previous layer and fitness of fuzzy variable for output $y^{5}$, while the output was the control rate $y^{5}$.

$$
y^{5}=\frac{\sum_{j=1}^{5}\left(m_{j} \sigma_{j}\right) y_{j}^{4}}{\sum \sigma_{j} y_{j}^{4}}
$$

where $m_{j}$ and $\sigma_{j}$ were the mean and standard of Gaussian membership function for output fuzzy sets, respectively.

Back-propagation algorithm was adopted in learning algorithm of the fuzzy neural network, with learning algorithm of parameters as follows:

$$
\begin{gathered}
m_{i j}(k+1)=m_{i j}(k)-\eta \frac{\partial E}{\partial m_{i j}} \\
\sigma_{i j}(k+1)=\sigma_{i j}(k)-\eta \frac{\partial E}{\partial \sigma_{i j}}
\end{gathered}
$$

where $\eta$ was learning rate of adjustable parameters.

Supposing expected density ramp was $\rho_{d}^{\prime}$; influence factor of ramp $\lambda$; length of ramp $l$; then error function was defined as:

$$
E_{k}=\frac{1}{2}\left[\left(\frac{y^{5}(k)-\rho_{d}}{\rho_{d}}\right)^{2}+\lambda\left(\frac{\frac{y^{5}(k)}{l}-\rho_{d}^{\prime}}{\rho_{d}^{\prime}}\right)^{2}\right]
$$

\section{CASE ANALYSis}

In the work, urbanized segments of Beijing-TianjinTanggu Expressway were selected as a case to validate the model. Due to limited detectors for actual sections, turning traffic volume was calculated through the product of detected traffic volume and distributing rate in selected sections, under the assumption that interchange distributing rate was constant. Thus, obtained traffic states of ramps were, used as input data for validation of the model. The traffic data of segments in Beijing-Tianjin-Tanggu Expressway in the direction towards Beijing were selected to validate the model, using MATLAB for simulation. Microscopic behaviors of vehicles and drivers were not considered in the algorithm. The evaluation on the model was mainly conducted from road traffic states.

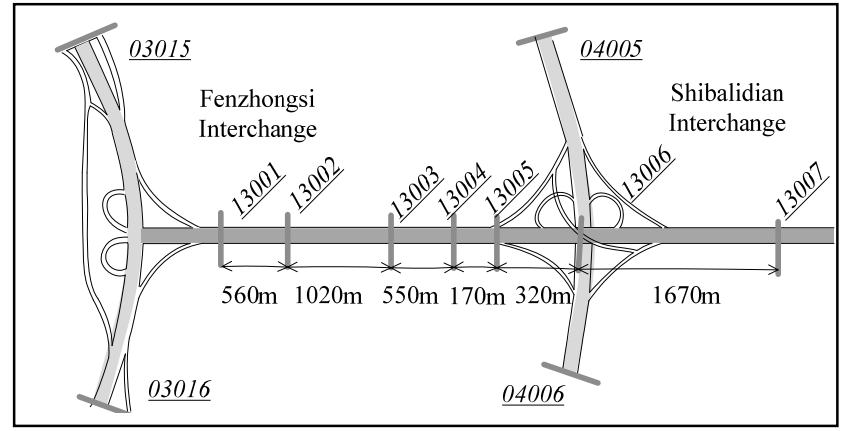

Figure 3. Research area of this paper

In order to visually present parameter changes of periphery traffic flow under or without effect of control, a two-dimensional spectrum was adopted to represent the state of traffic changes. Colors in Fig. 4-7 showed different traffic conditions (density and velocity); the 1-8 scores on the right coordinate represented expressway section 13001, 13002, 13003, 13004, 13005, 13006, 13007. The left coordinate of Fig. 4 and Fig. 5 represented traffic density; different colors indicated different traffic densities. The left coordinate of Fig. 6 and Fig. 7 showed traffic velocity; different colors represented different sizes of traffic velocity. According to comparison between Fig. 4 and Fig. 5, the density of expressway section 13002, 13006 was significantly improved through cooperative control, while the density of section 13003, 13004, 13007 declined in the 35th period. 
From comparison between Fig. 6 and Fig. 7, the velocity of section 13001 and 13006 was significantly improved through cooperative control, especially for section 13001.

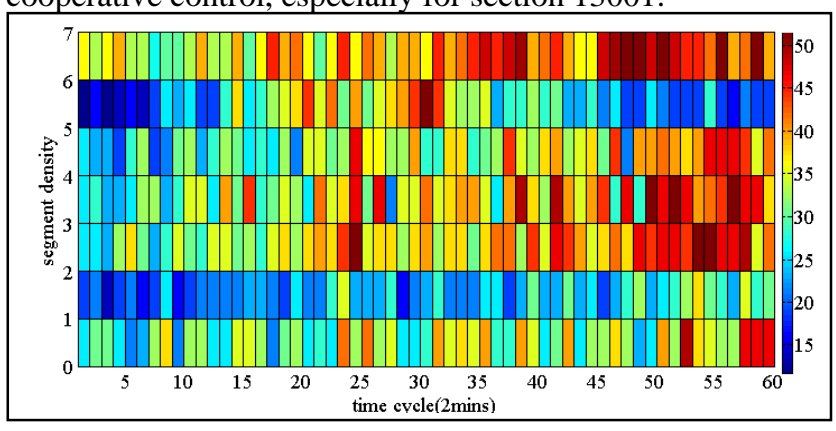

Figure 4. Series of traffic density of junction of road network without control (2min/cycle)

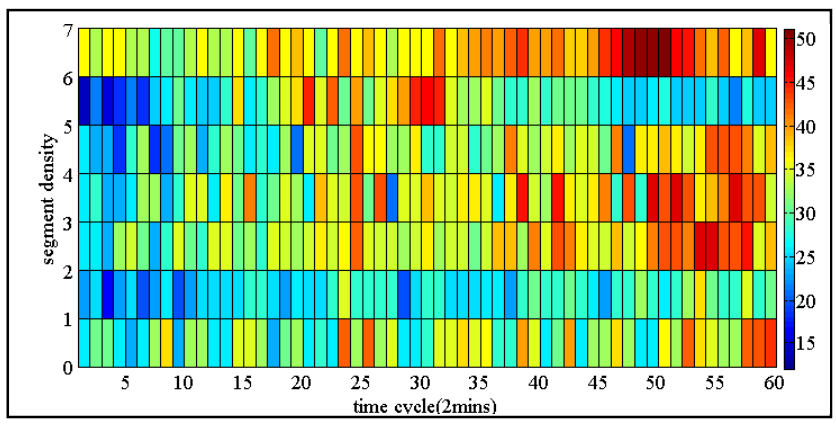

Figure 5. Series of traffic density of junction of road network with cooperative control

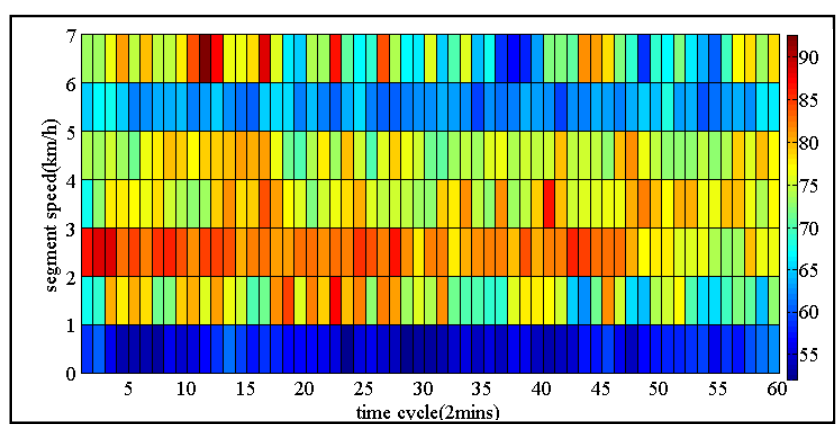

Figure 6. Series of traffic speed of junction of road network without control (2min/cycle)

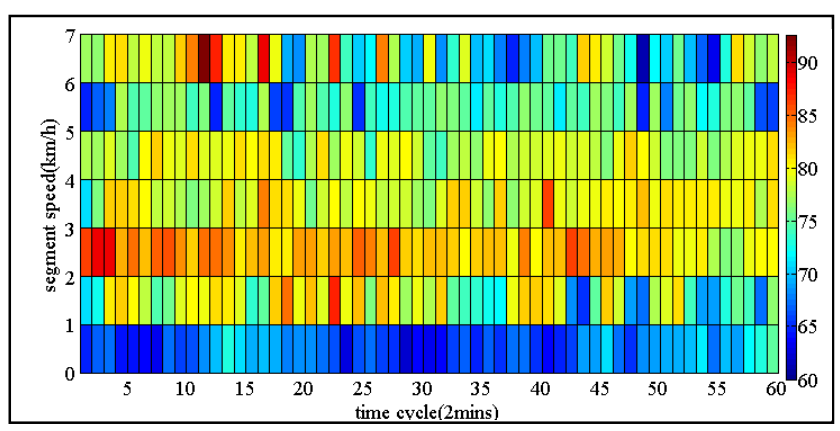

Figure 7. Series of traffic speed of junction of road network with cooperative control (2min/cycle)

\section{CONCLUSIONS}

Multi-agent ramp cooperative control method based on distributed network was researched in the work, based on multi-agent and fuzzy control theory. Experimental results show that the model achieves desirable control effect with fast response speed; traffic density of urbanized expressway is effectively stabilized, reducing traffic congestion in the area. Urban road traffic has nonlinearity, high dimensions and complex time-change. The work mainly studies cooperative control of multiple ramps in urbanized segments of expressway, without realization of cooperative control for the whole road network. Further study should be conducted for multi-ramp cooperative control of the whole road network for urban expressway.

\section{ACKNOWLEDGMENT}

The work is supported by The National Natural Science Foundation of China (71340020) under the title Research on Traffic Cooperative Control Method of the Junction of Isomerism Road Network.

\section{REFERENCES}

[1] M.Papageorgiou, A. Kotsialos, "Freeway ramp metering: An overview”. IEEE Transactions on Intelligent Transportation Systems, vol. 3, 2002, pp. 271-281, doi: 10.1109/TITS.2002.806803.

[2] A. Kotsialos, M. Papageorgiou, and M. Mangeas, "Coordinated and integrated control of motorway networks via non-linear optimal control”, Transportation Research Part C: Emerging Technologies, vol. 10, February, 2002, pp. 65-84, doi: 10.1016/S0968090X(01)00005-5.

[3] I. Papamichail, A. Kotsialos, and I. Margonis, "Coordinated ramp metering for freeway networks- A model-predictive hierarchical control approach", Transportation Research Part C: Emerging Technologies, vol. 18, June 2010, pp. 311-331, doi: 10.1016/j.trc.2008.11.002.

[4] J. Li, Y. H. Jia, X. B. Yao, “Coordinated networks flow control for interchange”, Journal of Beijng Jiaotong University, vol. 35, June, 2011, pp. 39-45, doi: 11-5258/U.20110311.1144.000.

[5] J. K. He, J. M. Xu, "Design of On-Ramp Controller Based on BP Neural Networks", Journal of South China University of Technology, vol. 30, July, 2002, pp. 24-27, doi: 10.3321/j.issn:1000565X.2002.07.007.

[6] X. P. Pan, G. Z. Zhang, "Neuro-fuzzy Network Control of Freeway On-Ramp Based on GA-BP”, Journal of Transportation Systems Engineering and Information Technology, vol. 8, April, 2008, pp. 7579, doi: 10.3969/j.issn.1009-6744.2008.02.012.

[7] G. Chai, X. Y. Gao, "Coordinated control method for freeway ramp with congestion of road section”, Journal of Southeast University (Natural Science Edition), vol. 43, May, 2013, pp. 654-658, doi: 10.3969/j.issn.1001-0505.2013.03.038.

[8] L. Wang, Z. J. Li, W. J. Xiu, "Freeway Off-ramp and Downstream Intersection Signal Coordination Based on MLD Model and MPC Approach", Control Engineering of China, vol. 21, July, 2014, pp. 487-490, doi: 10.3969/j.issn.1671-7848.2014.04.006.

[9] J. C. Rao, "Model and Control Method for area of the Freeway offramp”, Hangzhou, China: Zhejiang University, 2014.

[10] A. Fares, W. Gomaa, "Multi-agent reinforcement learning control for ramp metering". Advances in Intelligent Systems and Computing, vol. 330, 2014, pp. 167-173, doi: 10.1007/978-3-319-08422-0_25. 